\title{
Préservation et les possibilités d' usage les techniques modernes dans les principales cartothéques d' Estonie
}

\author{
by MARGIT TOHVER
}

Le problème le plus difficile en Estonie est qu' au cours de l' histoire, des collections de cartes ont été créés auprès des institutions très différentes aussi bien en ce qui concerne leur structure que leurs fonctions. Par conséquent, l' organisation, la description des documents, les conditions d' utilisation ainsi que l' encadrement matériel sont très variés. En gros, il est possible de diviser les collections en deux groupes:

1. Les archives, dont l' utilisation a été jusqu' à ces dernières années très limitée et dans lesquelles les cartes ont été rangées avant tout sur la base des noms de personnes. Dans ces collections, il n' existe pratiquement pas de fichiers, ou les fichiers viennent d' être créé seulement pendant les dernières années et très souvent $d$ ' une façon très originale. Pour avoir un aperçu du contenu de ces collections, il faudra tout d' abord cataloguer les matériaux. Les collections de cartes les plus importantes de ce genre se trouvent aux:

- Musée de l' Histoire d' Estonie, qui possède environ 2500 cartes et atlas de periode dès 17 . à 20 . siècle;

- Archives de l' Histoire d' Estonie, qui possède entre 80000 et 100000 cartes de même periode;

- Archives de la Direction territoriale d' Estonie, qui a en ce moment environ 70000 cartes et plans contemporaines;

- Archives de l' Etat d' Estonie qui possède des cartes dès 1918 jusqu' à aujourdhui.

A présent, on a commencé de cataloguer les cartes sur la base des recommandations de l' ISBD (CM) aux Archives de la Direction territoriale d' Estonie at aux Archives de l' Histoire d' Estonie. 
2. Le deuxième grand groupe est constitué par les collections de cartes des bibliothèques scientifiques. En 1996, les sept bibliothèques les plus importantes d' Estonie ont pris la décision de se servir du système intégré des données INNOPAC et de faire usage d' USMARC. Cette décision concerne directement les quatre collections des cartes les plus importantes en Estonie:

- la Bibliothèque de l' Université de Tartu

- la Bibliothèque des Archives du Musée de la Littérature d'Estonie

- la section cartographique de la Bibliothèque Nationale d' Estonie

Des fichiers électroniques existent en partie à la Bibliothèque Nationale d' Estonie et à la Bibliothèque de l' Université de Tartu. Toutefois, on utilise encore largement des fichiers manuels dans les bibliothèques et les données sont enregistrées en même temps dans des fichiers manuels et électronique. À la mise en marche de l' INNOPAC, les bases de données déjà existantes sont transférées en USMARC.

\section{Preservation et Restauration}

En moyenne $20 \%$ des documents de toutes les collections nécessitent des soins de restauration. A la Bibliothèque nationale d' Estonie et à la Bibliothèque de l' Université de Tartu, l' atelier de restauration des documents en feuille de grand format existe depuis longtemps. Quelques techniciens travaillent actuellement aussi dans les Archives de l' Histoire d' Estonie et au Musée de l' Histoire d' Estonie. On s' occupe surtout d' entoillage et restauration des grands formats sur des cadres horizontaux amovibles et aux Archives de l' Histoire d' Estonie de restauration des calques manuscrits.

La plus grande partie des magasins des cartothéques sont très petits, et par conséquent les documents se trouvent trop à l'étroit. Un autre problème est le grand nombre d' étagères en bois ainsi que l' utilisation des matériaux mal adaptés pour la restauration pendant les époques précédentes, ce qui expose les documents aux dommages biologiques et chimiques. Etant donné le niveau assez élémentaire des possibilités techniques de reproduction et de numérisation dans toutes les collections, la totalité des cartes est continuellement en utilisation et surtout les documents anciens n' arrêtent pas de subir des dommages physiques. Les dépenses sur la restauration sont très grandes partout, mais le pourcentage des matériaux nécessitant des soins ne diminue 
Préservation et les possibilités d' usage les techniques modernes dans les principales cartothéques d' Estonie

pas. En ce moment, il n'y a que la cartothèque de la Direction territoriale d' Estonie qui dispose des locaux et de l' ameublement adaptés.

Margit Tohver

National Library of Estonia

Tonismagi 2

EE0100-Tallinn, Estonia

Margitt@venus.nlib.ee 